\title{
Effective heuristics and meta-heuristics for the quadratic assignment problem with tuned parameters and analytical comparisons
}

\author{
Mahdi Bashiri ${ }^{*}$ and Hossein Karimi
}

\begin{abstract}
Quadratic assignment problem (QAP) is a well-known problem in the facility location and layout. It belongs to the NP-complete class. There are many heuristic and meta-heuristic methods, which are presented for QAP in the literature. In this paper, we applied 2-opt, greedy 2-opt, 3-opt, greedy 3-opt, and VNZ as heuristic methods and tabu search (TS), simulated annealing, and particle swarm optimization as meta-heuristic methods for the QAP. This research is dedicated to compare the relative percentage deviation of these solution qualities from the best known solution which is introduced in QAPLIB. Furthermore, a tuning method is applied for meta-heuristic parameters. Results indicate that TS is the best in 31\% of QAPs, and the IFLS method, which is in the literature, is the best in 58 $\%$ of QAPs; these two methods are the same in $11 \%$ of test problems. Also, TS has a better computational time among heuristic and meta-heuristic methods.
\end{abstract}

Keywords: Quadratic assignment problem, Heuristics, Meta-heuristics, Tuning method

\section{Background}

Quadratic assignment problem (QAP) is a well-known problem which delineates assigning number of economic facility to the same number of location. The objective function of this problem needs to be minimized. This problem was originally initiated by Koopmans and Beckmann (1957). QAP is used in combinational optimization, participating with traveling salesman problem (TSP) and graph problem. QAP is a kind of location problem and, additionally, a kind of layout problem. A lot of research presented exact solutions for QAP in the literature. Figure 1 shows the number of research, which applies the exact methods for QAP until 2005 (Loiola et al. 2007). These methods include branch and bound, dynamic programming, cutting plan, and branch and cut.

Sahni and Gonzalez (1976) proved that QAP belongs to the NP-complete class. In this class of the problem, computational time for solving problems, which have large instances, is too much and grows exponentially, so heuristic approaches and meta-heuristic methods are recommended

\footnotetext{
*Correspondence: Bashiri@shahed.ac.ir

Department of Industrial Engineering, Shahed University, Tehran 3319118651 , Iran
}

for solving this problem. Figure 2 shows the literature about solving QAP with heuristic and meta-heuristic methods. This figure reveals that the meta-heuristic methods have been applied more than the heuristic approaches.

For the first time, a simulated annealing (SA) approach in solving QAP was proposed by Burkard and Rendl (1984). Wilhelm and Ward (1987) then presented the new equilibrium components for solving QAP with SA, and after them, research suggested better SA solution methods. Skorin-Kapov (1990) executed the tabu search (TS) technique for QAP. After that, Taillard (1991) demonstrated robust TS for QAP. TS was also proposed for QAP in later research. Particle swarm optimization (PSO) is a new approach to solve QAP that Kennedy and Eberhart (1997), Shi et al. (2004), and Hongbo et al. presented (Hongbo and Ajith 2007; Hongbo et al. 2007). Figure 3 shows the application of meta-heuristic methods for QAP until 2005 (Loiola et al. 2007).

Figure 3 shows that many research used hybrid algorithm for QAP in recent years. Table 1 shows some of the recent research that applied TS, SA, and PSO for QAP from 2007 to 2009.

This paper is organized as follows: in the next section, QAP formulation is displayed; afterwards, the heuristic 


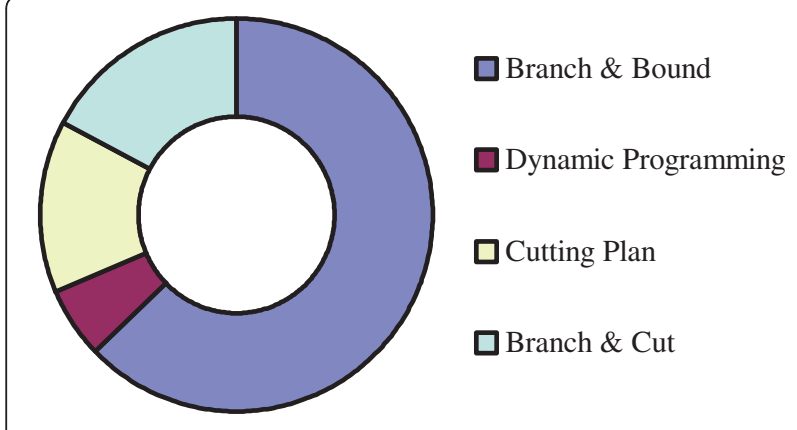

Figure 1 Exact methods for QAP.

methods are described. After that, the meta-heuristic methods such as SA, TS, and PSO are presented; the tuning method is described next. Computational analyses and comparison results are mentioned in the 'Results and discussion' section, and the last section is the 'Conclusions' section.

\section{Methods}

\section{QAP formulation}

This section is dedicated to introduce the classical formulation of QAP considered in this research. This formulation is as follows:

$$
\begin{aligned}
& \min \sum_{i=1}^{n} \sum_{j=1}^{n} \sum_{k=1}^{n} \sum_{l=1}^{n} c_{i j k l} x_{i k} x_{j l}, \\
& \sum_{i=1}^{n} x_{i j}, \forall j=1 \ldots n, \\
& \sum_{i=1}^{n} x_{i j}, \forall i=1 \ldots n, \\
& x_{i j}=\{0,1\}, \forall i, j=1 \ldots n,
\end{aligned}
$$

where $c_{i j k l}$ is the cost of assigning facility $i$ in location $k$ and simultaneously facility $j$ in location $l$, and $x_{i k}=1$ if location $k$ is assigned to facility $i$; otherwise, $x_{i k}=0$. Also, $x_{j l}=1$ if location $l$ is assigned to facility $j$; otherwise, $x_{j l}=0$. The objective function (Equation 1 ) of this model must be minimized. Each location must be assigned just to one facility, as Equation 2 shows. Equation 3 displays that each facility must be assigned just in

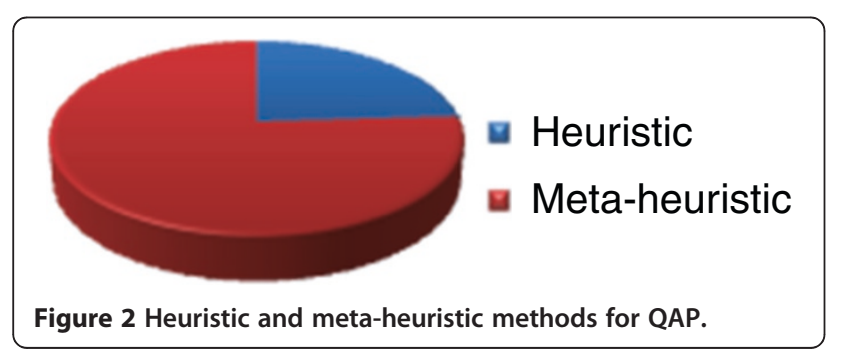

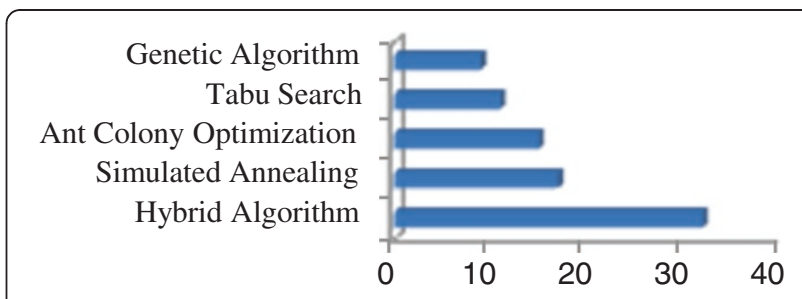

Figure 3 Meta-heuristic approach for QAP.

one location. The number of facility and location is the same and is equal to $n$. The variable in this model is binary.

\section{Heuristic methods}

Heuristic algorithms do not provide an assurance for optimization of the problem. These methods are an approximation. They have an additional property that worst-case solutions are known. In this section, some heuristic methods as procedures to search the better solution that contains 2-opt, greedy 2-opt, 3-opt, greedy 3-opt, and Vollman, Nugent, Zartler (VNZ) are contemplated.

\section{2-Opt algorithm}

Among simple local search algorithms, the most famous ones are 2-opt and 3-opt. The 2-opt algorithm was first proposed by Croes (1958) for TSP. If there are four locations and four facilities, the transposition of facility location in 2-opt method is like that in Figure 4. This figure illustrates that for the first transposition, the facility in location one can be changed with the facility in location two, and for the second transposition, the facility in location one can be changed with the facility in location three, so that if the number of location and facility is shown by $n$, the number of transposition in each iteration will be $n(n-1) / 2$.

Initially, the algorithm considers the transposition of facilities 1 and 2 . If the resulting solution's objective function value (OFV) is smaller than that of the initial solution, then it is stored as a candidate for future

Table 1 TS, SA, and PSO approach for QAP from 2007 to 2009

\begin{tabular}{llll}
\hline Authors & \multicolumn{3}{l}{ Meta-heuristic } \\
\cline { 2 - 4 } & TS & SA & PSO \\
\hline Hongbo and Ajith (2007); Hongbo et al. (2007) & - & - & . \\
Singh and Sharma (2008) & - & . & - \\
Zhu et al. (2009a,b); James et al. (James et al. 2007, & - & - & - \\
James et al. 2009); Fescioglu and Kokar (2008) & & & \\
This research & . & . & .
\end{tabular}

Dot (.), the authors used the method; hyphen (-), the authors did not use the method. 


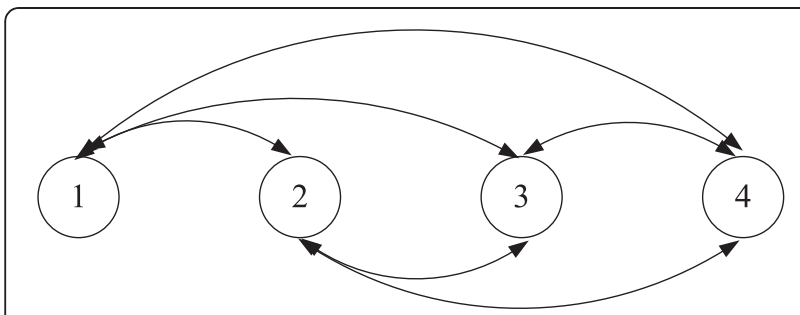

Figure 4 Transposition of facility in the 2-opt method.

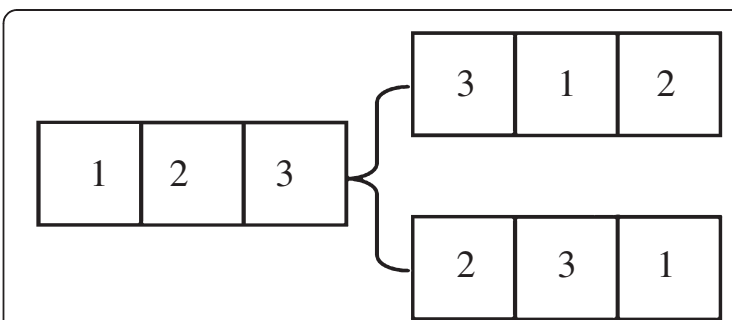

(1)

Figure 5 Types of transposition in 3-opt algorithm. consideration. Otherwise, it is discarded, and the algorithm considers the transposing of facilities 1 and 3 . If this exchange generates a better solution, then it is stored as a candidate for future consideration; otherwise, it is discarded, and so on. Thus, whenever a better solution is found, the algorithm discards the previous best solution. This procedure continues until all the pair-wise exchanges are considered.

For $n$ location in the QAP problem, the 2-opt algorithm consists of three steps:

Step 1. Let $S$ be the initial feasible solution and $Z$ its objective function value; then, set $S^{*}=S, Z^{*}=Z, i=$ 1 and $j=i+1=2$.

Step 2. Consider the exchange results in a solution $S^{\prime}$ that has OFV $Z^{\prime}<Z^{*}$, set $Z^{*}=Z^{\prime}$ and $S^{*}=S^{\prime}$. If $j<n$, then repeat step 2 ; otherwise, set $i=i+1$ and $j=i+1$. If $i<n$, repeat step 2 ; otherwise, proceed to step 3 .

Step 3. If $S \neq S^{*}$, set $S=S^{*}, Z=Z^{*}, i=1, j=i+1=2$ and go to step 2. Otherwise, output $S^{*}$ is selected as the best solution, and the process is terminated.

\section{Greedy 2-opt algorithm}

The greedy 2-opt algorithm is a variant of the 2-opt algorithm. The difference between this method and 2-opt is in selecting the best transposition. This method transposes the facility location if the OFV is better than the known OFV and stabilizes this assignment; it then goes to transpose the facility location from the start. It also consists of three steps. Like the 2-opt algorithm, greedy 2-opt also considers pair-wise exchanges. Initially, it considers transposing facilities 1 and 2 . If the resulting OFV is less than the previous one, two facilities are immediately transposed. Otherwise, the algorithm will go on to facility 3 and evaluate the exchange, and so on until improvement is found. If facilities 1 and 2 are transposed, then the algorithm will take it as an initial solution and will repeat the algorithm until it is impossible to improve the solution any further. Greedy 2-opt algorithm makes the exchange permanent whenever an improvement is found and thus consumes less computational time than the 2-opt algorithm. On the other hand, greedy 2-opt algorithm produces slightly worse solutions than the 2-opt algorithm.

\section{3-Opt algorithm}

The 3-opt algorithm is similar to the 2-opt algorithm except that it considers transposing two facilities at a time. This algorithm is originally applied for TSP by Bock (1958). For example, if there are three facilities in the same location, two types of transposition can be used with the 3-opt algorithm. These types are shown in Figure 5. Type (2) is applied in this research.

\section{Greedy 3-opt algorithm}

Greedy 3-opt algorithm is also similar to the greedy 2-opt algorithm, but it makes the three facility exchange permanent whenever its resulting OFV is better than the current OFV and repeats the algorithm with the new transposition as the initial solution. The transposition in this method is similar to that in 3-opt.

\section{VNZ method}

The VNZ method was introduced by Vollman et al. (1968). This method is using less storage space than 2-opt.

There is not any randomization, and also, these methods cannot orient the current solution to the optimum solution in a limited time. However, the meta-heuristic methods contain a good search approach with a reasonable time. These methods are considered in the next subsection.

\section{Meta-heuristic methods}

In the original definition, meta-heuristics are solution methods that manage an interaction between the local improvement procedures and higher level strategies to create a process capable of escaping from local optimum solution and performing a good search of solution space. These methods have also come to include any procedures that employ strategies for overcoming the trap of local optimality in complex solution spaces, especially those procedures that take advantage of one or more neighborhood structures as a means of defining acceptable moves to transformation from one solution to another. In this research, TS, SA, and PSO are applied for the QAP, and their comparison has been done for the selected data sets. 


\section{Tabu search}

Classical methods often face great difficulty when confronted with hard optimization problems present in real situations. Tabu search (TS), for the first time, was proposed by Glover $(1989,1990)$. This meta-heuristic approach is, in a theatrical manner, changing the ability of solving problems of practical significance. The pseudo code of TS, which is applied in this research, is as follows:

Step 1. Let $S$ be the initial feasible solution and $Z$ its objective function value; then, set $S^{*}=S, Z^{*}=Z$, max short-term memory $(\mathrm{STM})=5$, and $\max$ iteration $=1,000$; iter $=1$. Best $O$ value $=O$ value .

Step 2 . Random $(\underline{i}, j)=$ rand/Long-term memory (LTM) $(i, j),(n 1, n 2)=$ the indices of maximum value in random.

Step 3. If there is none $(n 1, n 2)$ in STM matrix, change $n 1$ and $n 2$ locations; otherwise, repeat step 2 .

Step 4. Insert $n 1$ and $n 2$ in STM and release the last indices from STM (e.g., $m 1, m 2)$; and $\operatorname{LTM}(m 1, m 2)$ $=\operatorname{LTM}(m 1, m 2)+1$.

Step 5. Calculate the objective function value $(Z)$ of the new permutation.

Step 6. If $Z \leq Z^{*}$, then $Z^{*}=Z, S^{*}=S$, and iter $=$ iter +1 .

Step 7. If iter $\leq \max$ iteration, then repeat step 2; otherwise, print $Z^{*}$ and $S^{*}$.

\section{Simulated annealing}

Simulated annealing is a famous and popular local search meta-heuristic applied to address discrete and continuous optimization problems. This method, like the other meta-heuristic methods, can be escaping from the local solution. Simulated annealing is so named because of its similarity to the process of physical annealing with solids, in which a crystalline solid is heated and then allowed to cool very slowly until it achieves its

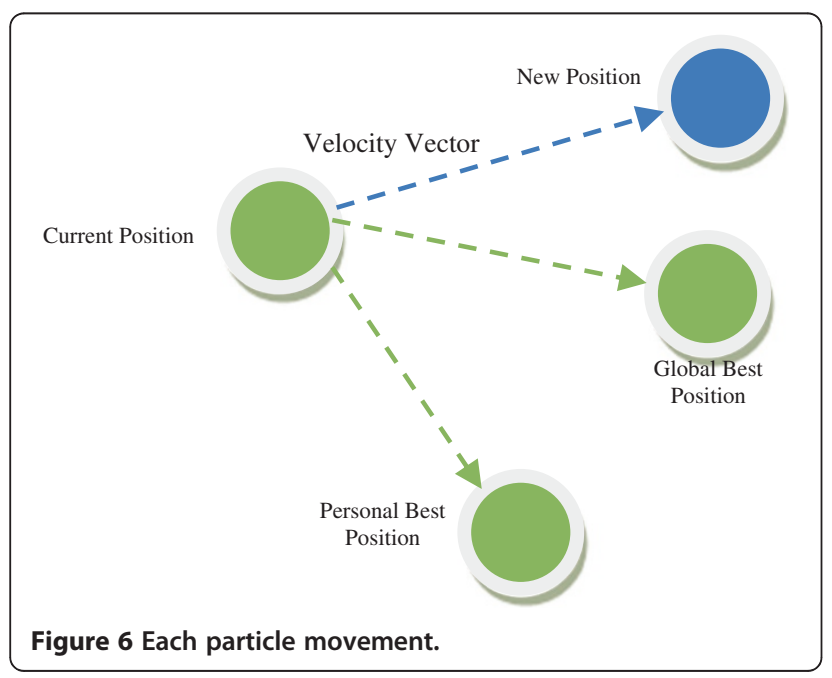

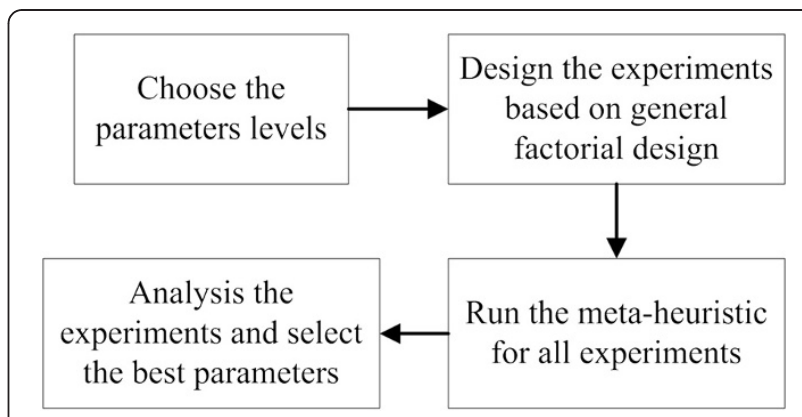

Figure 7 The procedure of tuning the parameters.

most regular possible crystal lattice configuration and thus is free of crystal defects. If the cooling schedule is sufficiently slow, the final configuration results in a solid with such superior structural integrity. Equation 5 shows the Metropolis acceptance criteria for each move in the cooling process (Metropolis et al. 1953).

$$
P=\exp \left(\frac{\left(\mathrm{OFV}_{B}-\mathrm{OFV}\right)}{T}\right),
$$

where $\mathrm{OFV}$ and $\mathrm{OFV}_{B}$ are the objective function values for this iteration and are the best computed one until this iteration. $T$ is the temperature of the algorithm in the iteration, and $P$ is the probability of acceptance for each move in the annealing process. The proposed SA pseudo code for QAP is as follows:

Step 1. Let $S$ be the initial feasible solution and $Z$ its objective function value; then, set $S^{*}=S, Z^{*}=Z$. $T=100, T_{0}=0.1, r=0.95$, nlimit $\max =5$ and nover $\max =10$.

Step 2. $n$ limit $=0$ and nover $=0$.

Step 3. Transpose two facilities in the current layout randomly and calculate the objective function value $(Z)$.

Step 4. If $Z \leq Z^{*}$, then accept the transposition; it means that $Z^{*}=Z, S^{*}=S$, and then $n$ limit $=$ nlimit +1 , nover $=$ nover +1 ; if nover $=$ nover $\max$ or $n$ limit $=$

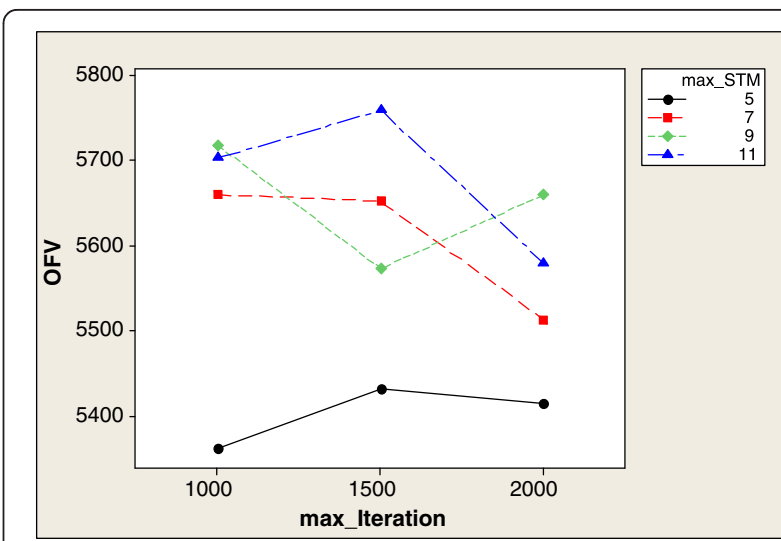

Figure 8 Interaction plot for OFV of 'Nug27'. 
nlimit max, then proceed to step 6.Otherwise, repeat step3; if $Z>Z^{*}$, then proceed to step 5.

Step 5. Calculate Equation 3; if $P \geq \operatorname{rand}(0,1)$, then $Z^{*}=$ $Z, S^{*}=S$, and then $n$ limit $=$ nlimit +1, nover $=$ nover +1 ; if nover $=$ nover max or $n$ limit $=n$ limit $\max$, then proceed to step 6; otherwise, repeat step 3.If $P<$ rand $(0,1)$, then nover $=$ nover +1 ; if nover $=$ nover $\max$, then proceed to step 6; otherwise, repeat step 3.

Step 6. $T=r \times T$, where $r$ is the rate of cooling. If $T \leq$ $T_{0}$, then proceed to step 7; otherwise, repeat step 2 .

Step 7. Print $S^{*}$ and $Z^{*}$.

\section{Partial swarm optimization}

The particle swarm optimization (PSO) is a populationbased search algorithm founded on the simulation of the social behavior of bees, birds, or a school of fish. This method is a significant member of the swarm intelligence. It was proposed by Eberhart and Kennedy as an optimization method (Eberhart and Kennedy 1995; Kennedy and Eberhart 1995). Each individual within the swarm is represented by a vector in multidimensional search space. This vector has one assigned vector that determines the next movement of the particle and is called the velocity vector. This technique also determines how to update the velocity of a particle. Each particle updates its velocity based on the current velocity and the best position (pbest) it has explored so far, as well as based on the global best position (gbest) explored by a swarm. Movement of each particle is shown in Figure 6, and it is based on Equations 6 and 7. Equation 6 illustrates that the velocity vector is updated by the global best position, personal best position, and current position of each particle. Equation 7 shows that each particle moves by its velocity.

$$
\begin{aligned}
v_{i}(t+1)= & w \cdot v_{i}(t)+b_{1} \cdot \operatorname{rand}\left(p \text { best }-x_{i}(t)\right) \\
& +b_{2} \cdot \operatorname{rand}\left(g \text { best }-x_{i}(t)\right), \\
x_{i}(t+1)= & x_{i}(t)+v_{i}(t),
\end{aligned}
$$

where $i$ is the index of the particle, $t$ is the index of an iteration, $v_{i}$ is the vector of velocity, $x_{i}$ is the position, $w$ is the weight of current velocity, $b_{1}$ is the weight of difference between personal best and current positions, $b_{2}$ is the weight of difference between global best and current

\begin{tabular}{|c|c|c|c|c|c|c|c|c|c|c|c|c|}
\hline \multirow{2}{*}{$\begin{array}{l}\text { Names of } \\
\text { instances }\end{array}$} & \multirow[t]{2}{*}{$n$} & \multicolumn{2}{|c|}{ 2-Opt } & \multicolumn{2}{|c|}{ Greedy 2-opt } & \multicolumn{2}{|c|}{ 3-Opt } & \multicolumn{2}{|c|}{ Greedy 3-opt } & \multicolumn{2}{|c|}{ VNZ } & \multirow{2}{*}{$\begin{array}{c}\text { Exact } \\
\text { Best OFV }\end{array}$} \\
\hline & & Best OFV & Time (s) & Best OFV & Time (s) & Best OFV & Time (s) & Best OFV & Time (s) & Best OFV & Time (s) & \\
\hline Nug12 & 12 & 630 & 0.035 & 720 & 0.070 & 612 & 0.060 & 662 & 0.038 & 630 & 0.115 & $\overline{578}$ \\
\hline Nug14 & 14 & 1040 & 0.033 & 1270 & 0.031 & 1076 & 0.120 & 1122 & 0.037 & 1094 & 0.079 & 1014 \\
\hline Nug15 & 15 & 1168 & 0.080 & 1410 & 0.031 & 1194 & 0.158 & 1266 & 0.081 & 1210 & 0.094 & 1150 \\
\hline Nug16a & 16 & 1636 & 0.036 & 2032 & 0.061 & 1710 & 0.150 & 1744 & 0.092 & 1708 & 0.160 & 1610 \\
\hline Nug16b & 16 & 1312 & 0.062 & 1478 & 0.039 & 1304 & 0.175 & 1342 & 0.047 & 1338 & 0.092 & 1240 \\
\hline Nug17 & 17 & 1764 & 0.043 & 2204 & 0.051 & 1824 & 0.183 & 1900 & 0.052 & 1812 & 0.094 & 1732 \\
\hline Nug18 & 18 & 1988 & 0.033 & 2438 & 0.038 & 2002 & 0.325 & 2102 & 0.078 & 2084 & 0.104 & 1930 \\
\hline Nug20 & 20 & 2676 & 0.095 & 3070 & 0.037 & 2700 & 0.300 & 2780 & 0.089 & 2762 & 0.076 & 2570 \\
\hline Nug21 & 21 & 2560 & 0.055 & 3224 & 0.073 & 2528 & 0.482 & 2612 & 0.145 & 2568 & 0.096 & 2438 \\
\hline Nug22 & 22 & 3836 & 0.050 & 4580 & 0.043 & 3706 & 0.702 & 3850 & 0.070 & 4002 & 0.172 & 3596 \\
\hline Nug24 & 24 & 3670 & 0.051 & 4550 & 0.052 & 3580 & 0.889 & 3776 & 0.121 & 3826 & 0.143 & 3488 \\
\hline Nug25 & 25 & 3816 & 0.056 & 4746 & 0.053 & 3850 & 0.999 & 4014 & 0.194 & 3946 & 0.186 & 3744 \\
\hline Nug27 & 27 & 5582 & 0.069 & 6436 & 0.037 & 5622 & 1.580 & 5932 & 0.214 & 5792 & 0.138 & 5234 \\
\hline Nug30 & 30 & 6180 & 0.081 & 7450 & 0.052 & 6282 & 3.476 & 6670 & 0.260 & 6648 & 0.238 & 6124 \\
\hline Bur26a & 26 & 5536606 & 0.104 & 5686514 & 0.079 & 5450887 & 5.318 & 5499462 & 0.390 & 5642823 & 0.269 & 5426670 \\
\hline Bur26b & 26 & 3865109 & 0.201 & 4078658 & 0.082 & 3825543 & 6.888 & 3921950 & 0.390 & 3898585 & 0.340 & 3817852 \\
\hline ur $26 c$ & 26 & 5559892 & 0.158 & 5691003 & 0.089 & 5432628 & 6.066 & 5488940 & 0.377 & 5560520 & 0.300 & 5426795 \\
\hline Bur26d & 26 & 3955005 & 0.160 & 4032499 & 0.079 & 3822905 & 5.472 & 3845665 & 0.408 & 3999274 & 0.324 & 3821225 \\
\hline Bur26e & 26 & 5457341 & 0.131 & 5656466 & 0.082 & 5387936 & 6.510 & 5441312 & 0.382 & 5610566 & 0.351 & 5386879 \\
\hline Bur26f & 26 & 3882393 & 0.149 & 4038741 & 0.079 & 3783547 & 4.828 & 3818354 & 0.390 & 3982126 & 0.282 & 3782044 \\
\hline Bur26g & 26 & 10178342 & 0.148 & 10668053 & 0.080 & 10119845 & 5.332 & 10243826 & 0.389 & 10405839 & 0.281 & 10117172 \\
\hline Bur26h & 26 & 7246369 & 0.101 & 7644484 & 0.085 & 7100009 & 5.651 & 7216120 & 0.391 & 7286571 & 0.281 & 7098658 \\
\hline
\end{tabular}
positions, and rand is used for randomization. The PSO

Table 2 Heuristic computational results for the test problems 
Table 3 Solution qualities with CPU time of small-size test problems

\begin{tabular}{|c|c|c|c|c|c|c|c|c|c|c|}
\hline \multirow{2}{*}{$\begin{array}{l}\text { Names of } \\
\text { instances }\end{array}$} & \multirow[t]{2}{*}{$n$} & \multicolumn{2}{|c|}{ SA } & \multicolumn{2}{|c|}{ TS } & \multicolumn{2}{|c|}{ PSO } & \multicolumn{2}{|c|}{ IFLS with local search (OXPM) } & \multirow{2}{*}{$\begin{array}{l}\text { Exact } \\
\text { OFV }\end{array}$} \\
\hline & & RPD & Time (s) & RPD & Time (s) & RPD & Time (s) & RPD & Time (s) & \\
\hline Nug12 & 12 & 0.00 & 0.16 & 0.00 & 0.12 & 7.61 & 0.27 & 1.38 & 0.70 & 578 \\
\hline Nug14 & 14 & 1.78 & 0.19 & 0.39 & 0.17 & 1.38 & 0.29 & 1.58 & 1.28 & 1,014 \\
\hline Nug15 & 15 & 3.30 & 0.16 & 0.87 & 0.15 & 2.26 & 0.27 & 0.17 & 1.69 & 1,150 \\
\hline Nug16a & 16 & 0.75 & 0.16 & 1.37 & 0.14 & 0.75 & 0.31 & 0.00 & 2.17 & 1,610 \\
\hline Nug16b & 16 & 0.65 & 0.17 & 0.00 & 0.14 & 0.00 & 0.32 & 0.00 & 2.14 & 1,240 \\
\hline Nug17 & 17 & 1.04 & 0.17 & 0.69 & 0.15 & 3.00 & 0.32 & 1.27 & 2.77 & 1,732 \\
\hline Nug18 & 18 & 2.69 & 0.18 & 1.04 & 0.14 & 3.01 & 0.34 & 0.93 & 3.42 & 1,930 \\
\hline Nug20 & 20 & 2.49 & 0.20 & 1.56 & 0.16 & 1.95 & 0.36 & 0.70 & 5.28 & 2,570 \\
\hline Nug21 & 21 & 1.72 & 0.20 & 0.25 & 0.17 & 2.71 & 0.40 & 0.25 & 6.89 & 2,438 \\
\hline Nug22 & 22 & 1.78 & 0.20 & 0.50 & 0.17 & 1.33 & 0.41 & 1.33 & 7.77 & 3,596 \\
\hline Nug24 & 24 & 4.59 & 0.21 & 1.15 & 0.18 & 3.96 & 0.45 & 2.87 & 10.91 & 3,488 \\
\hline
\end{tabular}

pseudo code, which is presented for QAP in this research, is as follows:

Step 1. $\max$ iteration $=100$, number of particle $=15$, and $w=n-1, b_{1}=n / 2$ and $b_{2}=(n / 2)+2$, where $n$ is the dimension of the problem. Make 15 permutations as the initial solutions and $Z^{*}=\min$ $(\mathrm{OFV}), S^{*}=S$, and iter $=1$.

Step 2. For $i=1$ to $\mathrm{w}$, transpose two facility. Do this step for each particle.
Step 3. Calculate objective function value $(Z)$ for each new particle; find the personal best OFV for each particle ( $p$ best), and find the global best OFV (gbest).

Step 4. Generate a random discrete number between 0 and $b_{1}$, and for $i=1$ to this random number, simulate each particle to $p$ best.

Step 5. Generate a random discrete number between 0 and $b_{2}$, and for $i=1$ to this random number, simulate each particle to gbest.

Table 4 Solution qualities with CPU time of medium-size test problems

\begin{tabular}{|c|c|c|c|c|c|c|c|c|c|c|}
\hline \multirow{2}{*}{$\begin{array}{l}\text { Names of } \\
\text { instances }\end{array}$} & \multirow[t]{2}{*}{$n$} & \multicolumn{2}{|c|}{ SA } & \multicolumn{2}{|c|}{ TS } & \multicolumn{2}{|c|}{ PSO } & \multicolumn{2}{|c|}{ IFLS with local search (OXPM) } & \multirow{2}{*}{$\begin{array}{c}\text { Exact } \\
\text { OFV }\end{array}$} \\
\hline & & RPD & Time (s) & RPD & Time (s) & RPD & Time (s) & RPD & Time (s) & \\
\hline Nug25 & 25 & 3.69 & 0.24 & 1.55 & 0.18 & 1.92 & 0.48 & 0.21 & 12.97 & 3,744 \\
\hline Bur26a & 26 & 0.10 & 0.62 & 0.09 & 0.53 & 0.20 & 1.32 & 0.09 & 15.86 & $5,426,670$ \\
\hline Bur26b & 26 & 0.67 & 0.61 & 0.19 & 0.54 & 0.42 & 1.30 & 0.17 & 15.56 & $3,817,852$ \\
\hline Bur26c & 26 & 0.06 & 0.60 & 0.26 & 0.55 & 0.30 & 1.34 & 0.00 & 15.41 & $5,426,795$ \\
\hline Bur26d & 26 & 0.12 & 0.60 & 0.02 & 0.54 & 0.05 & 1.30 & 0.01 & 15.38 & $3,821,225$ \\
\hline Bur26e & 26 & 0.01 & 0.60 & 0.03 & 0.51 & 0.05 & 1.33 & 0.26 & 15.16 & $5,386,879$ \\
\hline Bur26f & 26 & 0.26 & 0.61 & 0.05 & 0.54 & 0.33 & 1.29 & 0.00 & 15.55 & $3,782,044$ \\
\hline Bur26g & 26 & 0.06 & 0.59 & 0.01 & 0.54 & 0.42 & 1.34 & 0.01 & 15.28 & $10,117,172$ \\
\hline Bur26h & 26 & 0.03 & 0.60 & 0.01 & 0.52 & 0.35 & 1.32 & 0.00 & 14.88 & $7,098,658$ \\
\hline Nug27 & 27 & 2.67 & 0.26 & 1.38 & 0.22 & 4.43 & 0.48 & 2.79 & 17.42 & 5,234 \\
\hline Nug30 & 30 & 5.94 & 0.27 & 2.65 & 0.22 & 6.01 & 0.54 & 1.32 & 22.45 & 6,124 \\
\hline Tai30a & 30 & 4.70 & 0.27 & 4.75 & 0.25 & 5.90 & 0.85 & 2.46 & 18.28 & $1,818,146$ \\
\hline Tai30b & 30 & 3.55 & 0.26 & 1.62 & 0.25 & 5.18 & 0.87 & 2.33 & 18.23 & $637,117,113$ \\
\hline Tai40a & 40 & 5.31 & 0.37 & 6.12 & 0.32 & 5.53 & 1.16 & 3.16 & 62.66 & $3,139,370$ \\
\hline Tai40b & 40 & 5.00 & 0.34 & 3.07 & 0.29 & 3.84 & 1.07 & 4.18 & 60.44 & $637,250,948$ \\
\hline Tai50a & 50 & 5.90 & 0.47 & 6.49 & 0.39 & 7.19 & 1.36 & 2.90 & 158.94 & $4,938,796$ \\
\hline Tai50b & 50 & 3.86 & 0.45 & 5.15 & 0.40 & 7.23 & 1.35 & 1.87 & 161.27 & $458,821,517$ \\
\hline
\end{tabular}


Table 5 Solution qualities with CPU time of large size test problems

\begin{tabular}{|c|c|c|c|c|c|c|c|c|c|c|}
\hline \multirow{2}{*}{$\begin{array}{l}\text { Names of } \\
\text { instances }\end{array}$} & \multirow[t]{2}{*}{$n$} & \multicolumn{2}{|c|}{ SA } & \multicolumn{2}{|c|}{ TS } & \multicolumn{2}{|c|}{ PSO } & \multicolumn{2}{|c|}{ IFLS with local search (OXPM) } & \multirow{2}{*}{$\begin{array}{c}\text { Exact } \\
\text { OFV }\end{array}$} \\
\hline & & RPD & Time (s) & RPD & Time $(s)$ & RPD & Time (s) & RPD & Time (s) & \\
\hline Lipa80a & 80 & 0.25 & 0.89 & 0.15 & 0.79 & 40.70 & 2.39 & 0.72 & 1734.11 & 253,195 \\
\hline Lipa80b & 80 & 23.46 & 0.94 & 23.55 & 0.80 & 23.93 & 2.38 & 20.65 & 1889.81 & $7,763,962$ \\
\hline Tai80a & 80 & 6.13 & 0.94 & 6.66 & 0.79 & 7.01 & 2.37 & 2.87 & 1390.58 & $13,527,910$ \\
\hline Tai80b & 80 & 2.93 & 0.96 & 3.91 & 0.82 & 4.55 & 2.48 & 0.71 & 1122.56 & $841,223,593$ \\
\hline Lipa90a & 90 & 2.01 & 1.12 & 0.49 & 0.93 & 1.03 & 3.07 & 0.67 & 2964.41 & 360,630 \\
\hline Lipa90b & 90 & 13.55 & 1.17 & 14.59 & 0.96 & 14.71 & 3.35 & 0.00 & 3200.88 & $12,490,441$ \\
\hline Tai100a & 100 & 4.66 & 1.69 & 4.11 & 1.20 & 4.33 & 4.82 & 2.69 & 3560.03 & $21,090,402$ \\
\hline Tai100b & 100 & 3.06 & 2.15 & 2.02 & 1.73 & 4.17 & 5.21 & 0.93 & 3595.17 & $1.186 \mathrm{E}+09$ \\
\hline
\end{tabular}

Step 6. iter $=$ iter +1 ; if iter $<\max$ iteration, then repeat step 2;otherwise, proceed to step 7.

Step 7. Print $Z^{*}=$ gbest $S^{*}=$ gbest permutation $(S)$.

\section{Tuning method}

The parameters in meta-heuristic methods which are introduced, such as max STM and max iteration, were tuned by carrying out the general factorial design. To achieving this purpose, some levels were defined initially for each parameter. These levels are determined to be focused on the logic of meta-heuristics. For instance, when the number of iteration in PSO is increased, then the time of search is intensified. This is just one characteristic of this meta-heuristic approach. After defining the levels, an experimental design is determined by general factorial design. The meta-heuristic for these experiments is then run, and their results are analyzed. Thus, the best parameter level can be found. Figure 7 illustrates this tuning method.

For example, for 'Nug' instances taken from the Quadratic Assignment Problem Library (QAPLIB), the analysis is done for 'Nug27' for the TS method. The proposed levels for max STM are 5, 7, 9, and 11, and for max iteration, they are $1,000,1,500$, and 2,000. We consider two replicates for each combination of factor levels; hence, for this instance, 24 treatment combinations are arranged. Figure 8 shows the interaction plot for OFV, and it also shows the best combination of these factors. The best max STM is 5, and the best max iteration is 1,000 . Hence, we tune the TS parameter based on this experiment. Moreover, the parameters like nover max, nlimit max, $T, T_{O}$, and others were set by analyzing the general factorial design similar to TS.

\section{Results and discussion}

\section{Computational analyses}

In this research, an analytical comparison between heuristics and meta-heuristics, which are described as the Nug and 'Bur' instances selected from QAPLIB, are applied. The computer which carried out the experiments is equipped with a $253-\mathrm{GHz}$ processor with programming coded in MATLAB7.8 (MathWorks, Natick, MA, USA). Table 2 shows the computational results for heuristic methods. Results show that 3-opt as a heuristic method is the best solution method for QAP among the presented heuristic methods in this research. In this table, sizes of the problems are determined by $n$.

Tables 3, 4, and 5 reveal the computational results for meta-heuristic methods. In this computational analysis, the test problems are classified in small-, medium-, and large-size cases. These groups are taken from the study of Ramkumar et al. (2008). Our experiment results are compared with iterated fast local search (IFLS) when the order crossover with random pair-wise interchange mutation (OXPM) is used (Ramkumar et al 2008). The criterion considered for evaluating the performance is the relative percentage deviation (RPD) of the solution quality from the best known solution. The number of location is $n$.

The superior methods in CPU time and OFV are illustrated in the boldface. The average of gaps for heuristic methods and meta-heuristic methods introduced in this research with the exact solution are shown in Figure 9. These results are related to the Nug and Bur instances.

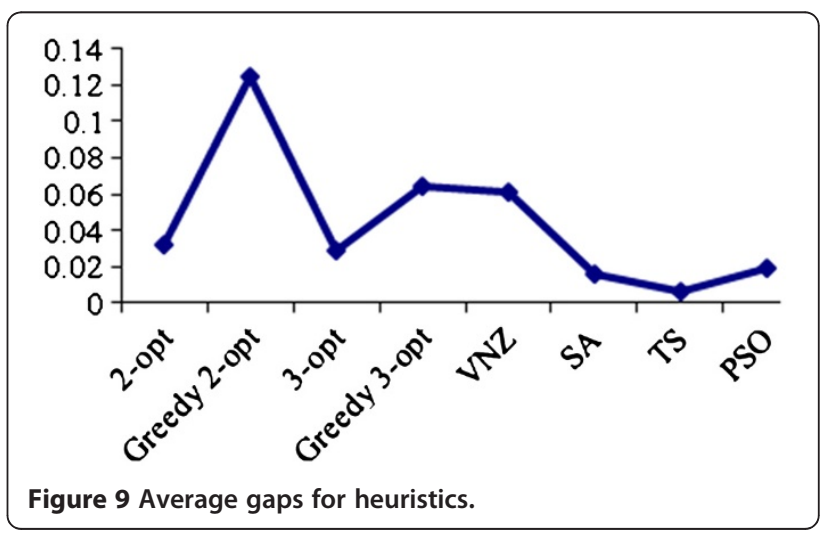



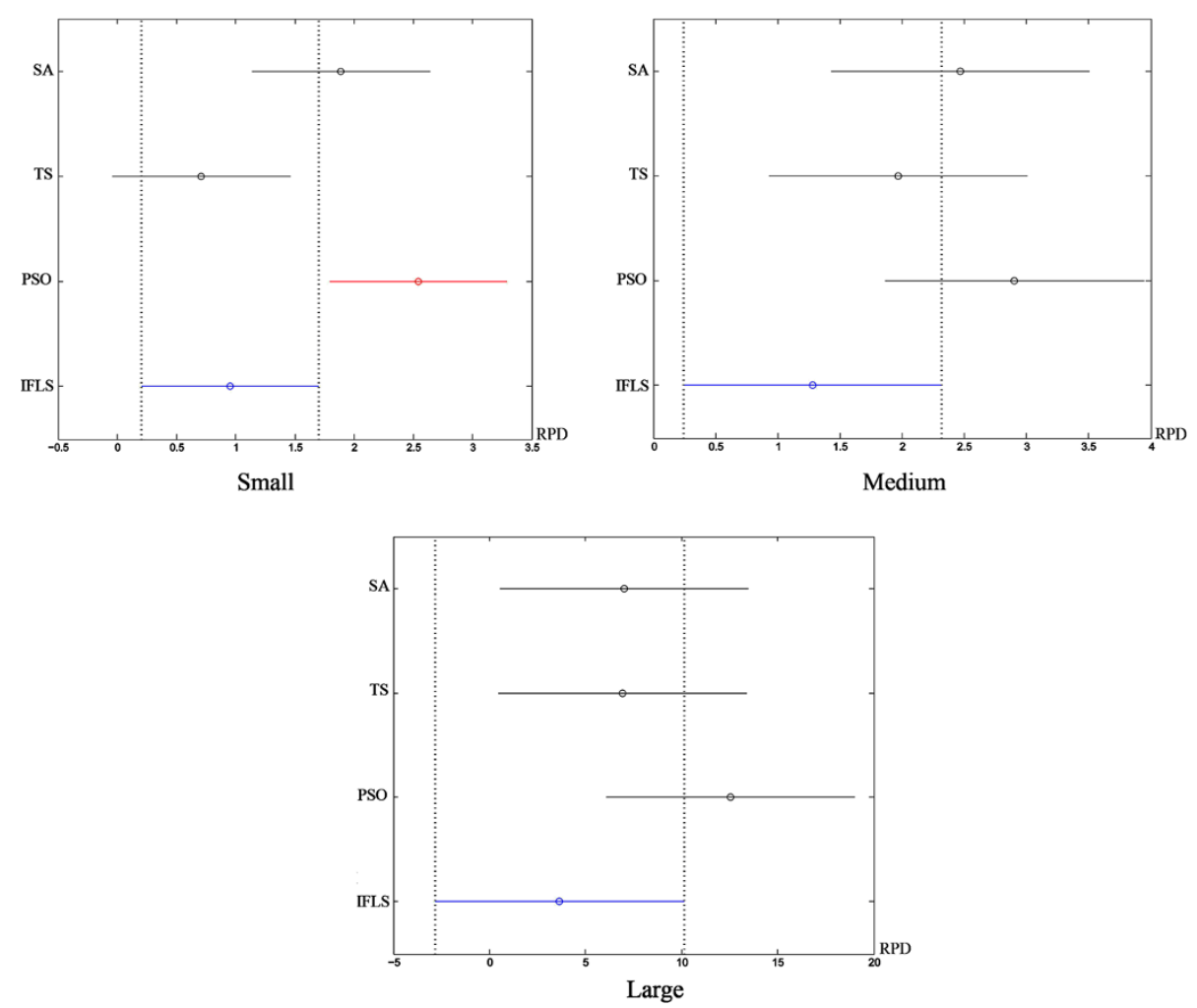

Figure 10 ANOVA results give $95 \%$ confidence interval for the difference among meta-heuristics and IFLS.

This figure shows that TS is the best in our methods. In addition, it can be concluded that meta-heuristics have better solution quality; hence, we compare metaheuristic methods with IFLS. Furthermore, Figure 9 shows that the greedy-2opt is not a good method because the average gap for this method is about $12 \%$ in selected data sets.

The performances of our proposed meta-heuristic approaches and IFLS with local search are studied by analysis of variance (ANOVA) test.

Figure 10 summarizes the results of our investigation where the critical value for this analysis is considered as 0.05. As we can observe from Figure 10, in small-size test problems, the means of IFLS and PSO are significantly different, and no meta-heuristics have means significantly different from IFLS in the medium- and largetest problems. It is clear from this figure that TS and IFLS have the best solution quality in small-, medium-, and large-size instances, respectively. Hence, it is not necessary that the $p$ values of these analyses be declared.

\section{Conclusions}

This research considers the heuristic and meta-heuristic solution methods for QAP, and the comparison among them has been done. In addition, a tuning method is declared. The comparison has been executed for the selected data set which was extracted from QAPLIB. The results show that 3-opt has better results than the other heuristic methods. Moreover, our meta-heuristic methods are better than the heuristic methods in solution quality. In this paper, some small, medium, and large test problems are used for comparing our metaheuristic methods to a method from literature (i.e., IFLS with local search). ANOVA test is run for the results, and it showed that our methods are not considerably different from IFLS. TS is the most excellent method in computational time. Comparisons between the selected solution methods for more instances from the QAPLIB and comparisons of these algorithms with other metaheuristics and hybrid algorithms can be conducted in future research.

\section{Competing interests}

The authors declare that they have no competing interests.

\section{Authors' contributions}

MB has worked on the literature and modeling; he also proposed the TS, SA, and PSO for his work. HK has performed numerical experiments and carried out sensitivity analysis. Both authors read and approved the final manuscript.

\section{Acknowledgments}

The authors would like to thank the anonymous referees for their constructive comments on earlier version of this work.

Received: 22 May 2010 Accepted: 3 March 2012

Published: 18 July 2012 


\section{References}

Bock F (1958) An algorithm for solving traveling-salesman and related network optimization. Research report associated with talk presented at the Operations Research Society of America 14th National Meeting, St. Louis

Burkard R, Rendl F (1984) A thermodynamically motivated simulation procedure for combinatorial optimization problems. European Journal of Operational Research 17:169-174

Croes GA (1958) A method for solving traveling salesman problems. Operation Research 6:791-812

Eberhart RC, Kennedy J (1995) A new optimizer using particle swarm theory. Paper presented at the Proceedings of the Sixth International Symposium on Micro Machine and Human Science, Nagoya, Japan

Fescioglu U, Kokar M (2008) Application of Self Controlling Software Approach to Reactive Tabu Search. SASO, Venezia, pp 297-305

Glover F (1989) Tabu search: Part I. ORSA J Comput 1:190-206

Glover F (1990) Tabu search: Part II. ORSA J Comput 2:4-32

Hongbo L, Ajith A (2007) An hybrid fuzzy variable neighborhood particle swarm optimization algorithm for solving quadratic assignment problems. Jucs 13 (9):1309-1331

Hongbo L, Ajith A, Jianying Z (2007) A particle swarm approach to quadratic assignment problems. Soft Comput Ind App 39:213-222

James T, Rego C, Glover F (2007) A cooperative parallel tabu search algorithm for the quadratic assignment problem. Eur J Oper Res 195:810-826

James T, Rego C, Glover F (2009) Multistart tabu search and diversification strategies for the quadratic assignment problem. Systems, Man and Cybernetics, Part A: Systems and Humans 39(3):579-596

Kennedy J, Eberhart RC (1995) Particle swarm optimization. In: Proceedings of IEEE International Conference on Neural Networks, Nov-Dec 1995, 4th edn. IEEE, Piscataway, pp 1942-1948

Kennedy J, Eberhart RC (1997) A discrete binary version of the particle swarm algorithm. In: Proceedings of International Conference on Systems, Man, and Cybernetics, October 1994, 1st edn. IEEE Computer Society Press, New York, pp 4104-4108

Koopmans T, Beckmann M (1957) Assignment problems and the location of economic activities. Econometrica 25:53-76

Loiola E, de Abreo N, Boaventura-Nett P, Hahn P, Querido T (2007) A survey for the quadratic assignment problem. Eur J Oper Res 176:657-690

Metropolis N, Rosenbluth A, Rosenbluth M, Teller A, Teller M (1953) Equation of state calculations for fast computing machines. Journal of Chemical 21: 1087-1092

Ramkumar AS, Ponnambalam SG, Jawahar N, Suresh RK (2008) Iterated fast local search algorithm for solving quadratic assignment problems. Rob ComputIntegr Manuf 24:392-401

Sahni S, Gonzalez T (1976) P-complete approximation problems. J Assoc Comput Mach 23:555-565

Shi X, Xing X, Wang Q (2004) A discrete PSO method for generalized TSP problem. In: Proceedings of International Conference on Machine Learning and Cybernetics, August 2004, 4th edn. IEEE Computer Society Press, New York, pp 2378-2383

Singh SP, Sharma RK (2008) Two-level modified simulated annealing based approach for solving facility layout problem. Int J Prod Res 46(13):3563-3582

Skorin-Kapov J (1990) Tabu search applied to the quadratic assignment problem. ORSA J Comput 2:33-45

Taillard E (1991) Robust taboo search for the quadratic assignment problem. Parallel Comput 17:443-455

Vollman TE, Nugent CE, Zartler RL (1968) A computerized model for office layout. $J$ Ind Eng 19:321-327

Wilhelm MR, Ward TL (1987) Solving quadratic assignment problems by simulated annealing. IEEE Trans 19:107-119

Zhu W, Curry J, Marquez A (2009a) GPU-accelerated simt tabu search for the quadratic assignment problem. NAMRC, Greenville, pp 435-442

Zhu W, Curry J, Marquez A (2009b) SIMD tabu search for the quadratic assignment problem with graphics hardware acceleration. Int J Prod Res 48:1035-1047

doi:10.1186/2251-712X-8-6

Cite this article as: Bashiri and Karimi: Effective heuristics and metaheuristics for the quadratic assignment problem with tuned parameters and analytical comparisons. Journal of Industrial Engineering International 2012 8:6.

\section{Submit your manuscript to a SpringerOpen ${ }^{\circ}$ journal and benefit from:}

- Convenient online submission

- Rigorous peer review

- Immediate publication on acceptance

- Open access: articles freely available online

- High visibility within the field

- Retaining the copyright to your article

Submit your next manuscript at $>$ springeropen.com 